Canad. Math. Bull. Vol. 21 (3), 1978

\title{
DIMENSION THEORY VIA REDUCED BISECTOR CHAINS
}

\author{
BY \\ LUDVIK JANOS
}

\begin{abstract}
Let $(X, d)$ be a metric space and $Y$ and $Z$ subsets of $X$. We say that $Z$ is a bisector in $Y$ and write $Y \triangleright Z$ iff $Y \supset Z$ and there are two distinct points $y_{1}, y_{2} \in Y$ such that $Z=\left\{z: d\left(z, y_{1}\right)=d\left(z, y_{2}\right)\right.$ and $\left.z \in Y\right\}$. By a reduced bisector chain in $(X, d)$ of length $n$ we understand a chain $X=$ $X_{0} \triangleright X_{1} \triangleright \cdots \triangleright X_{n-1} \triangleright X_{n}$ such that $\operatorname{dim} X_{n} \leq 0$ and $\operatorname{dim} X_{n-1}>0$. By $r(X, d)$ we denote the maximum length of reduced bisector chains in $(X, d)$. For a metrizable topological space $X$ we introduce the topological invariant $r(X)$ as the minimum of $r(X, d)$ taken over the set of all metrizations $d$ of $X$. We prove that the function $r(X)$ coincides with the dimension of $X$ on the class of compact metric spaces.
\end{abstract}

1. Introduction and notation. If $x_{1}, x_{2} \in X$ are two distinct points in a metric space $(X, d)$ we denote by $B\left(x_{1}, x_{2}\right)$ the bisector of $x_{1}, x_{2}$, i.e., the set $\left\{x: d\left(x, x_{1}\right)=d\left(x, x_{2}\right)\right\}$. If $Y$ is a subset of $(X, d)$ we say that $Y$ is a bisector in $(X, d)$ iff there are two distinct points $x_{1}, x_{2}$ in $X$ such that $Y=B\left(x_{1}, x_{2}\right)$. The relevancy of this concept to topological dimension, denoted in the sequel by $\operatorname{dim} X$, has been brought to light in our recent paper [3] where the following result is obtained.

THEOREM 1.1. If in a compact metric space $(X, d)$ every bisector has dimension $\leq n-1$ then $\operatorname{dim} X \leq n .(n=0,1, \ldots)$

This result depends heavily upon a theorem of J. Nagata (see [4] Theorem 11.2. page 18) and our observation that the family of open half-spaces of a compact metric space $(X, d)$ forms a subbasis for the topology of $X$. (see [3] Lemma 2.1.)

The inductive character of Theorem 1.1. calls naturally for the consideration of consecutive formation of bisectors. If $Y$ and $Z$ are subsets of a metric space

Received by the editors July 6, 1977.

AMS 1970 subject classifications:

primary $54 \mathrm{~F} 45$

Secondary 55 C 10

54 E 35

Key words and phrases:

Bisector, bisector-chain, dimension, metrization, topological invariant, expressability of a topological property in a suitable language. 
$(X, d)$ we say that $Z$ is a bisector of $Y$ and write $Y \triangleright Z$ iff $Y \supset Z$ and $Z$ is a bisector in $(Y, d)$ where $(Y, d)$ is the metric space induced by the metric $d$ on the subset $Y$. Thus we have defined the binary relation $\triangleright$ between subsets of $X$ which permits us to introduce a bisector chain $(b c)$ as a sequence $\left\{X_{i}\right\}_{o}^{n}$ of subsets of $X$ satisfying $X_{i} \triangleright X_{i+1}$ for $i=0,1, \ldots, n-1$, and shall write it in the form:

$$
X_{0} \triangleright X_{i} \triangleright \cdots \triangleright X_{n-1} \triangleright X_{n}
$$

In [3] we considered chains starting with $X$, i.e., $X=X_{0}$ and proceeding as far as possible, i.e., the terminal member $X_{n}$ was either a singleton or bisectorempty which means $X_{n} \triangleright \Theta$, where $\Theta$ denotes the empty set. At that time we were not aware of certain results due to J. H. Roberts [5] indicating the importance of $b c$ with at most zero-dimensional terminals.

Defintrion 1.1. A bisector chain $\left(^{*}\right)$ in a metric space $(X, d)$ is said to be a reduced bisector chain $(r b c)$ iff $X=X_{0}, \operatorname{dim} X_{n} \leq 0$ and $\operatorname{dim} X_{n-1}>0$. The integer $n$ is called the length of the $r b c$. The reduced bisector chain has length zero iff it is of the form $X=X_{0}$ where the metric space $(X, d)$ has dimension zero.

The question arises as to whether a metric space $(X, d)$ possesses a $r b c$. If $\operatorname{dim} X=0$, then, by the definition, the $b c X=X_{0}$ is the only $r b c$ in $(X, d)$ and its length is zero. Assume now $\operatorname{dim} X>0$. This implies that $X$ is an infinite set which in turn implies the existence of bisectors $B\left(x_{1}, x_{2}\right)$ in $X$. If for some $x_{1}, x_{2} \in X$ the bisector $B\left(x_{1}, x_{2}\right)$ is empty, i.e., $\operatorname{dim} B\left(x_{1}, x_{2}\right)=-1$, then the chain $X \triangleright \Theta$ is a $r b c$ of length 1 . If $B\left(x_{1}, x_{2}\right) \neq \Theta$ the dimension of $B\left(x_{1}, x_{2}\right)$ is either 0 , in which case the chain $X \triangleright B\left(x_{1}, x_{2}\right)$ is again a $r b c$ of length 1 , or the dimension of $B\left(x_{1}, x_{2}\right)$ is $>0$ and the process continues applying the above reasoning to $B\left(x_{1}, x_{2}\right)$. This means that if $\operatorname{dim} X>0$ three cases may be considered:

(1) there exists in $(X, d)$ an infinite chain $X=X_{0} \triangleright X_{1} \triangleright \cdots \triangleright X_{n} \triangleright \cdots$ with $\operatorname{dim} X_{n}>0$ for $n=0,1, \ldots$

(2) There exists in $(X, d)$ a $r b c$ of arbitrary large length.

(3) The length $n$ of $r b c$ 's in $(X, d)$ is bounded.

We now assign to every nonempty metric space $(X, d)$ a non-negative integer (or $\infty$ ) which we call the maximal length of $r b c$ in $(X, d)$ and denote it by $r(X, d)$ as follows:

(a) We set $r(X, d)=0$ iff $\operatorname{dim} X=0$.

(b) We set $r(X, d)=\max \{n$ :there exists a $r b c$ in $(X, d)$ of length $n\}$ iff $\operatorname{dim} X>0$ and case (3) takes place.

(c) We set $r(X, d)=\infty$ iff $\operatorname{dim} X>0$ and either case (1) or case (2) takes place.

For a metrizable topological space $X$ we introduce the topological invariant 
$r(X)$ as the minimum of $\{r(X, d): d \in M(X)\}$ where $M(X)$ denotes the set of all metrics on $X$ inducing the topology of $X$, or expressed in equivalent terms: $r(X)$ is the minimum of $r(Y, d)$, where $(Y, d)$ ranges through the class of metric spaces homeomorphic to $X$.

The purpose of this paper is to prove the following two statements.

TheOREM 1.2. The function $r(X)$ coincides with $\operatorname{dim} X$ on the class of compact metric spaces.

THEOREM 1.3. For the $n$-dimensional Euclidean space $E^{n}$ we have $r\left(E^{n}\right)=n$ for $n=1,2, \ldots$

\section{Relation between bisectors and the geometric theory of $\mathbf{J}$. H. Roberts.}

Lemma 2.1. Let $Y_{0} \triangleright Y_{1} \triangleright \cdots \triangleright Y_{n}$ be a bc in a metric space $(X, d)$. Then there exists a bc $X=X_{0} \triangleright X_{1} \triangleright \cdots \triangleright X_{n}$ in $(X, d)$ such that $Y_{i}=X_{i} \cap Y_{0}$ for $i=0,1, \ldots n$.

Proof. For $i=1,2, \ldots n, Y_{i}$ is a bisector in $Y_{i-1}$, hence there are two distinct points $y_{i-1}^{\prime}$ and $y_{i-1}^{\prime \prime}$ in $Y_{i-1}$ such that $Y_{i}=B\left(y_{i-1}^{\prime}, y_{i-1}^{\prime \prime}\right) \cap Y_{i-1}$.

Defining recursively $X_{1}=B\left(y_{0}^{\prime}, y_{0}^{\prime \prime}\right)$

and

$$
X_{2}=B\left(y_{1}^{\prime}, y_{1}^{\prime \prime}\right) \cap X_{1}
$$

$$
\dot{X}_{n}=B\left(y_{n-1}^{\prime}, y_{n-1}^{\prime \prime}\right) \cap X_{n-1}
$$

we obtain the chain of required properties.

Corollary 2.2. The function $r(X)$ is monotonic, i.e., if $Y$ is a nonempty subset of a metrizable topological space $X$ then we have $r(Y) \leq r(X)$.

Proof. Let $d \in M(X)$ be a metric on $X$ for which $r(X)=r(X, d)$. Assume now that the statement is false, i.e., $r(X)<r(Y)$. Since $r(Y) \leq r(Y, d)$ we obtain $r(X, d)<r(Y, d)$. The assumption $r(X)<r(Y)$ implies that $r(X)$ is finite, say $n \geq 0$. Thus, there exists in $(Y, d)$ a $b c \quad Y=Y_{0} \triangleright Y_{1} \triangleright \cdots \triangleright Y_{n} \triangleright Y_{n+1}$ for which $\operatorname{dim} Y_{n}>0$. Lemma 2.1. implies the existence of a $b c \quad X=$ $X_{0} \triangleright X_{1} \triangleright \cdots \triangleright X_{n} \triangleright X_{n+1}$ with $Y_{n}=X_{n} \cap Y_{0}$. Since $Y_{n} \subset X_{n}$ and the dimension function $\operatorname{dim} X$ is monotonic we have that $\operatorname{dim} X_{n}>0$ implying that $r(X, d)$ is at least $n+1$ contrary to our assumption.

In order to formulate the geometrical result of J. H. Roberts we need to make some trivial observations concerning the $b c$ in Euclidean spaces $E_{n}$.

LEMMA 2.3. Every bisector $Y$ in the $n$-dimensional Euclidean space $\left(E^{n}, e\right)$ $(n=1,2, \ldots)$ equipped with the Euclidean metric $e$ is a hyperplane, i.e., an affine subspace of $E^{n}$ of dimension $n-1$, and conversely every affine subspace of dimension $n-1$ is a bisector in $\left(E^{n}, e\right)$. 
Proof. If $x_{1}, x_{2} \in E^{n}$ and $x_{1} \neq x_{2}$, the bisector $B\left(x_{1}, x_{2}\right)$ can be defined as a hyperplane passing through the point $1 / 2\left(x_{1}+x_{2}\right)$ and orthogonal to the vector $x_{2}-x_{1}$; it is clear that every hyperplane can be obtained this way.

Corollary 2.4. If $E^{n}=Y_{0} \triangleright Y_{1} \triangleright \cdots \triangleright Y_{k}$ is $a$ bc in the Euclidean space $\left(E^{n}, e\right)(n=1,2, \ldots)$ then each member $Y_{i}$ is an affine subspace of dimension $n-i, i=1,2, \ldots, k$, and conversely, if $Y$ is an affine subset of $E^{n}$ of dimension $m(0 \leq m \leq n)$ then there exists a bc $E^{n}=Y_{0} \triangleright Y_{1} \triangleright \cdots \triangleright Y_{k}=Y$ of length $k=$ $n-m$ connecting $E^{n}$ and $Y$.

Proof. Straight-forward by induction on $K$.

THEOREM 2.5. Let $X$ be a nonempty subset of $\left(E^{2 n+1}, e\right)$ such that $\operatorname{dim}(X \cap$ $Y) \leq 0$ for every affine subset $Y$ of $E^{2 n+1}$ of dimension $n+1$. Then $r(X, e) \leq n$ where $(X, e)$ is the metric space induced on $X$ by the euclidean metric $e$.

Proof. Assume that $X=X_{0} \triangleright X_{1} \triangleright \cdots \triangleright X_{k}$ is an arbitrary $b c$ in $(X, e)$ such that $\operatorname{dim} X_{k}>0$. Lemma 2.1. implies that there is a $b c$ in $\left(E^{2 n+1}, e\right) E^{2 n+1}=$ $Y_{0} \triangleright Y_{1} \triangleright \cdots \triangleright Y_{k}$ with $X_{k}=Y_{k} \cap X$. Since $\operatorname{dim} X_{k}>0$ and $\operatorname{dim}(Y \cap X) \leq 0$ for every affine subset of dimension $n+1$, this implies that $\operatorname{dim} Y_{k}>n+1$. On the other hand we know that the dimension of $Y_{k}$ is precisely $2 n+1-k$, so that $k<n$ showing that no $r b c$ in $(X, e)$ can be longer than $n$.

We now confront this result with the theorem of J. H. Roberts ([5] Theorem 12).

THEOREM 2.6. If a separable metric space $X$ has dimension $n$ then there exists a topological embedding $f: X \rightarrow E^{2 n+1}$ such that $\operatorname{dim}(f(X) \cap Y) \leq 0$ for every affine subset $Y$ of $E^{2 n+1}$ of dimension $n+1$.

Corollary 2.7. If $X$ is a separable metrizable space then $r(X) \leq \operatorname{dim} X$.

Proof. If $\operatorname{dim} X=\infty$ there is nothing to prove, therefore assume $\operatorname{dim} X$ finite, say $n \geq 0$. Theorem 2.6. implies that a homeomorphic image of $X$, namely $f(X)$ satisfies the hypothesis of Theorem 2.5. furnishing $r(f(X), e) \leq n$ from which our assertion follows.

3. Proofs of Theorems 1.2. and 1.3. To prove Theorem 1.2. means to show that for every non-negative integer $k \geq 0$ we have

$$
r(X)=k \quad \text { if and only if } \operatorname{dim} X=k
$$

for every compact metrizable space $X$. We shall proceed by induction on $k$. For $k=0$ the statement $\left(^{* *}\right)$ is true by the very definition of $r(X)$. In order to carry out the induction step we need

LEMMA 3.1. Let $X$ be a metrizable topological space with $r(X)<\infty$, and assume that $Y \subset X$ is a bisector in $(X, d)$ where $d \in M(X)$ is such that $r(X)=$ $r(X, d)$. Then $r(Y)<r(X)$. 
Proof. The length of an $r b c$ in $(Y, d)$ is not greater than $r(X)-1$. Thus $r(Y, d)<r(X)$ and therefore $r(Y)<r(X)$.

Now assume that the validity of the statement $\left({ }^{* *}\right)$ has been established for all values $k=0,1, \ldots, n$ and assume

(a) $X$ compact and $r(X)=n+1$. Consider the metric space $(X, d)$ where $d$ is such that $r(X)=r(X, d)$. Lemma 3.1. implies that every bisector $Y$ in $(X, d)$ is such that $r(Y) \leq n$ which by the induction hypothesis yields that $\operatorname{dim} Y \leq n$ from which we conclude, using Theorem 1.1. that $\operatorname{dim} X \leq n+1$. Confronting this result with Corollary 2.7., we finally have $\operatorname{dim} X=n+1$, which proves one half of the statement. To prove the second half assume

(b) $X$ compact and $\operatorname{dim} X=n+1$. From Corollary 2.7. we know $r(X) \leq$ $n+1$. But if $r(X)<n+1$ then the induction hypothesis would yield $r(X)=$ $\operatorname{dim} X<n+1$ contrary to the assumption. Thus we have $r(X)=n+1$ and the proof of Theorem 1.2. is complete.

We now prove Theorem 1.3. as an easy corollary of Theorem 1.2. and the monotonic property of the function $r(X)$.

From Corollary 2.4. follows that $r\left(E^{n}, e\right)=n(n=1,2, \ldots)$ implying that $r\left(E^{n}\right) \leq n(n=1,2, \ldots)$.

Denoting by $I_{n}$ the unit cube in $E_{n}$ we obtain from Theorem 1.2. that $r\left(I^{n}\right)=n(n=1,2, \ldots)$ and since $I^{n} \subset E^{n}$ Corollary 2.2. yields finally $r\left(E^{n}\right)=$ $n(n=1,2, \ldots)$ what had to be shown.

4. Some logical aspects of our results. In our paper [3] we introduced the function $b(X, d)$ as the maximum length of $b c s$ in a metric space $(X, d)$ and the corresponding topological invariant $b(X)$ as the minimum of $\{b(X, d): d \epsilon$ $M(X)\}$.

Despite similarity between the definitions of $b(X, d)$ and $r(X, d)$ there is an essential difference between these notions from the point of view of formal logic and we need some definitions to bring this distinction to light.

Defintrion 4.1. For a nonempty metric space $(X, d)$ we introduce the ternary relation $R \subset X \times X \times X$ on $X$ setting $\left(x_{1}, x_{2}, x_{3}\right) \in R$ iff $x_{1} \neq x_{2}$ and $x_{3} \in B\left(x_{1}, x_{2}\right)$.

Remark 4.1. The relation $R$ is defined naturally by the concept of bisector $B\left(x_{1}, x_{2}\right)$. In the sequel we shall also deal with two quaternary relations on $X, I \subset X \times X \times X \times X$ and $E \subset X \times X \times X \times X$ defined on a metric space $(X, d)$ by $\left(x_{1}, x_{2}, x_{3}, x_{4}\right) \in I$ iff $d\left(x_{1}, x_{2}\right) \leq d\left(x_{3}, x_{4}\right)$ and $\left(x_{1}, x_{2}, x_{3}, x_{4}\right) \in E$ iff $d\left(x_{1}, x_{2}\right)=$ $d\left(x_{3}, x_{4}\right)$ respectively. It is obvious that $E$ can be expressed in terms of $I$ and $R$ in turn can be expressed in terms of $E$. We can say that the relations $R, E$ and $I$ introduce on $X$ the bisector-, equational- and inequality-structure, respectively. The corresponding languages which can talk about these structures shall be denoted by $L, L_{E}$ and $L_{I}$ respectively. 
Definition 4.2. Let $L$ denote the first order language containing besides the logical connectives, $\neg, \mathrm{V}, \wedge, \rightarrow, \exists$ and $\forall$ and variables $x_{1}, x_{2}, \ldots$ only one ternary predicate symbol $R^{*}$. If $P$ is a property of a metric space we say that $P$ is expressible in the language $L$ provided there is a sentence $S$ (i.e., a formula without free variables) in $L$ such that a metric space $(X, d)$ has the property $P$ if and only if $(X, d)$ is a model of $S$, assuming that the predicate symbol $R^{*}$ is interpreted by $R$ in $X$. The fact that $(X, d)$ is a model of $S$ will be denoted by $(X, d) \vDash S$.

Remark 4.2. Analogously we understand the expressability of $P$ in the language $L_{E}$ or $L_{I}$. Since $L$ can be conceived as a sublanguage of $L_{E}$ and $L_{E}$ as a sublanguage of $L_{I}$ the expressability in $L$ implies that in $L_{E}$ and consequently in $L_{\mathrm{I}}$.

THEOREM 4.1. The property $b(X, d)=n$ for $n=0,1, \ldots$ is expressible in $L$.

Proof. The sentence $S_{0}=\forall x_{1} \forall x_{2} \forall x_{3} \neg R^{*}\left(x_{1} x_{2} x_{3}\right)$ says precisely that every bisector in $(X, d)$ is empty. Applying this result to any bisector $B\left(x_{4}, x_{5}\right)$ of a metric space $(X, d)$ we can express the fact that $b(X, d) \leq 1$ by the formula $S_{1}^{\prime}=\forall x_{1} \forall x_{2} \forall x_{3} \forall x_{4} \forall x_{5}\left[R^{*}\left(x_{4} x_{5} x_{1}\right) \wedge R^{*}\left(x_{4} x_{5} x_{2}\right) \wedge R^{*}\left(x_{4} x_{5} x_{3}\right) \rightarrow \neg R^{*}\left(x_{1} x_{2} x_{3}\right]\right.$. Proceeding inductively we can produce formulas $S_{n}^{\prime}$ expressing the property $b(X, d) \vDash S_{n}(n=1,2, \ldots)$. Thus the statement $b(X, d)=0$ is equivalent to $(X, d) \vDash S_{0}$ and the statement $b(X, d)=n$ for $n=1,2, \ldots$ is equivalent to $(X, d) \vDash S_{n}$ where $S_{n}=S_{n}^{\prime} \wedge \neg S_{n-1}^{\prime}$ and where we set $S_{0}^{\prime}=S_{0}$.

It is clear that this statement is no longer true if we pass from the property $b(X, d)=n$ to the property $r(X, d)=n$ since the conditions $\operatorname{dim} X_{n} \leq 0$ and $\operatorname{dim} X_{n-1}>0$ involved in the definition of $r b c$ are not in any obvious way describable in terms of the relation $R$. This is the main reason why the results obtained in this paper cannot be considered as definite.

Definition 4.3. To each sentence $S$ in the language $L$ we assign the topological property $P_{s}$ defined on the class of metrizable spaces as follows. We say that a space $X$ has the topological property $P_{s}$ iff there is a metric $d \in M(X)$ for which $(X, d) \vDash S$, and we express this by saying that $P_{s}(X)$ is true.

Definition 4.4. Let $P$ be a topological property and $C$ a subclass of the class of metrizable spaces. We say that $P$ is expressible in the language $L$ on the class $C$ provided there are sentences $S_{1}, S_{2}, \ldots, S_{m}$ in $L$ and a formula $F\left(p_{1}, p_{2}, \ldots, p_{m}\right)$ of the sentential logic such that for $X \in C$ the truth value of $P(X)$ coincides with that of $F\left(P_{s_{1}}(X), P_{s_{1}}(X), \ldots P_{s_{m}}(X)\right]$.

TheORem 4.2. The topological property $b(X)=n$ for $n=0,1, \ldots$ is expressible in $L$ on the class of metrizable spaces. 
Proof. If $n=0$ the fact $b(X)=0$ means that there is $d \in M(X)$ with $(X, d)$ ₹ $S_{0}$ showing that the property $b(X)=0$ is expressible. Assume now $n>1$. In this case the fact $b(X)=n$ means that there is $d \in M(X)$ for which $b(X, d)=n$ but it is not true that there is such $d^{\prime} \in M(X)$ for which $b\left(X, d^{\prime}\right)=n-1$. Thus the statement $\left\{\left[d \in M(X)(x, d) \vDash S_{n}\right]\right.$ and not $\left.\left[d^{\prime} \in M(x)\left(X, d^{\prime}\right) \vDash S_{n-1}\right]\right\}$ is the desired expression of the property $b(x)=n$.

Corollary 4.3. The property $\operatorname{dim} X=0$ is expressible in $L$ on the class of compact metrizable spaces.

Proof. This follows readily from the above theorem and the basic result of our paper [3] where we proved that if $X$ is compact then $\operatorname{dim} X=0$ iff $b(X)=0$.

Our main conjecture is that for arbitrary $n \geq 0$ the property $\operatorname{dim} X=n$ is expressible in $L$ on the class of compact metrizable spaces.

Our belief in the truth of this conjecture is supported by a result of $\mathrm{J}$. de Groot (see [1] or [4] page 154, Corollary to Theorem V.5). This result can easily be translated in the language $L_{I}$ and it reads:

Theorem 4.4. (De Groot) The property $\operatorname{dim} X=n(n=0,1, \ldots)$ is expressible in the language $L_{I}$ on the class of compact metrizable spaces.

Analogously, our result [2] on the metric rigidity if translated in the equational language $L_{E}$ reads:

The property $\operatorname{dim} X=0$ is expressible in the language $L_{E}$ on the class of separable metrizable spaces.

\section{REFERENCES}

1. J. de Groot, On a metric that characterizes dimension, Can. J. Math. 9 (1957) 511-514.

2. L. Janos, A metric characterization of zero-dimensional spaces, Proc. Amer. Math. Soc., 31 (1972) 268-270.

3. L. Janos, Dimension theory via bisector chains, Canad. Math. Bull. 20 (1977), 313-317.

4. J. Nagata, Modern dimension theory, Interscience Publishers, New York, 1965.

5. J. H. Roberts, A theorem on dimension, Duke Math. J. 8 (1941), 565-574.

DEPARTMENT OF MATHEMATICS, MisSISSIPPI STATE UNIVERSITY,

MississiPPI STATE, MisSISSIPPI 39762 